\title{
Profile of netarsudil ophthalmic solution and its potential in the treatment of open-angle glaucoma: evidence to date
}

This article was published in the following Dove Press journal:

Clinical Ophthalmology

\section{Lily Dasso \\ Tala Al-Khaled \\ Sriram Sonty \\ Ahmad A Aref}

Department of Ophthalmology and Visual Sciences, Illinois Eye \& Ear Infirmary, University of Illinois at Chicago College of Medicine, Chicago, IL, USA
Correspondence: Sriram Sonty Department of Ophthalmology and Visual Sciences, Illinois Eye \& Ear Infirmary, University of Illinois at Chicago College of Medicine, 1700 East West Road,

Calumet City, IL 60409, USA

Tel +l 708 89| 3330

Email ssonty@msn.com

\begin{abstract}
Netarsudil ophthalmic solution is a novel topical intraocular pressure (IOP)-lowering agent that has recently been approved by the US Food and Drug Administration (FDA) for the treatment of ocular hypertension and open-angle glaucoma. Its unique pharmacology allows for IOP lowering as a result of direct reduction in trabecular outflow resistance in addition to a decrease in episcleral venous pressure and aqueous humor production. The efficacy of netarsudil has been shown in animal studies and human clinical trials. It has been shown to be noninferior to the therapy with topical timolol in individuals with baseline IOP $<25 \mathrm{mmHg}$. Importantly, netarsudil has been shown to reduce IOP to the same degree, regardless of baseline levels. There are no known systemic safety issues associated with netarsudil. The most common local adverse effects relate to conjunctival hyperemia. The once-daily dosing schedule is advantageous for individuals who have difficulties with medication adherence. Further studies of a combination of netarsudil and latanoprost agents are currently underway.
\end{abstract}

Keywords: glaucoma treatment, Rho kinase, intraocular pressure lowering, glaucoma medical therapy

\section{Introduction}

Glaucoma continues to be a leading cause of irreversible blindness currently affecting over 60 million people worldwide. ${ }^{1}$ The glaucoma population is estimated to increase to over 110 million by 2040, augmenting the need for new approaches to glaucoma therapy. ${ }^{1}$ Epidemiological studies have shown that the risk of open-angle glaucoma progression increases with a higher intraocular pressure (IOP) at an estimated 12\% increase in risk for every $1 \mathrm{mmHg}$ increase in IOP. ${ }^{2}$ Medical intervention that lowers IOP is currently the first-line therapy for preventing progression of optic nerve damage and subsequent vision loss. ${ }^{3}$

IOP is regulated by a balance between the production of aqueous humor by the ciliary processes and the rate of aqueous outflow to venous circulation through the uveoscleral outflow pathway and trabecular meshwork (TM) pathways. ${ }^{4}$ The majority of the aqueous humor is filtered through the TM pathway, also known as the conventional pathway, which consists of the TM, juxtacanalicular tissue (JCT), and Schlemm's canal (SC). ${ }^{5}$ Although the pathophysiology of glaucomatous degeneration is not completely understood, a resistance to aqueous outflow through the TM is thought to cause an increase in IOP and eventually lead to optic nerve damage. ${ }^{4}$

Currently available therapeutic options mainly increase uveoscleral outflow (known as outflow drugs) or decrease aqueous humor production (inflow drugs) in order to 
lower IOP instead of targeting the diseased TM pathway directly. ${ }^{6,7}$ Medications that increase the uveoscleral outflow include the prostaglandin analogs and alpha agonists, and medications that decrease the aqueous humor production include beta blockers, carbonic anhydrase inhibitors, and alpha agonists. ${ }^{7}$ Pilocarpine is a unique miotic glaucoma drug that works to increase aqueous humor outflow but does not directly target the TM. Rather, this agent induces contraction of the ciliary muscle in order to expand the TM and decrease outflow resistance. ${ }^{8}$ The present issue with the current medical options is that necessary IOP reductions cannot be achieved with monotherapy alone in many patient cases. ${ }^{9}$

Netarsudil (AR-13324) is a novel topical ocular hypotensive glaucoma medication recently approved by the US Food and Drug Administration (FDA) in December 2017. ${ }^{10}$ This review aims to assess the current research concerning this new medication and its potential to treat open-angle glaucoma and ocular hypertension.

\section{Netarsudil mechanism of action}

ROCK is a naturally occurring serine/threonine protein kinase that serves to promote assembly of actin stress fibers and focal adhesions within the TM. Netarsudil is a potent ROCK inhibitor that is an amino isoquinoline amide that also inhibits the norepinephrine transporter (NET). The agent is formulated with 2,4-dimethyl benzoate to improve bioavailability (Figure 1). ${ }^{11}$ Netarsudil has been shown to have three novel mechanisms of action that contribute to lowering IOP. Primarily, the agent functions to lower IOP directly through the relaxation of the TM and contraction of ciliary muscle, leading to an increase in aqueous outflow through the conventional pathway. ${ }^{5,12,13}$ ROCK1 and ROCK2

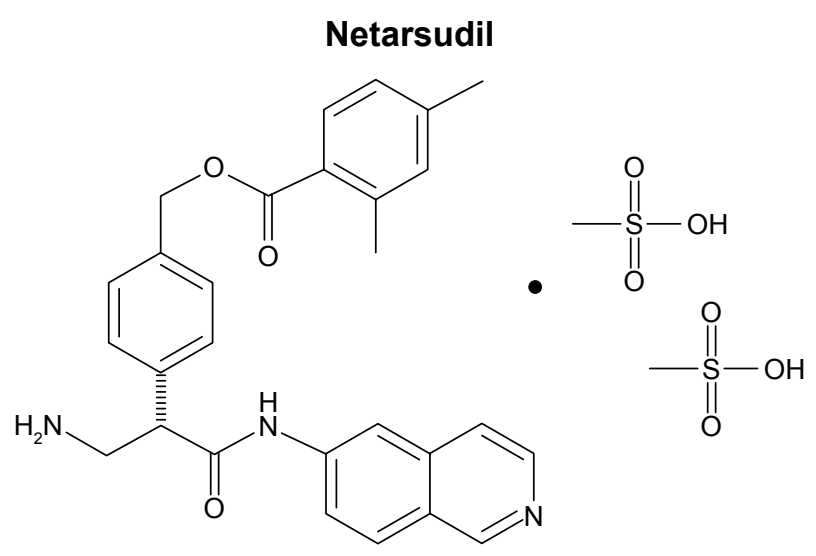

Figure I Netarsudil chemical structure depicting the free base and dimesylate to improve bioavailability.

Note: Reproduced with permission from Aerie Pharmaceuticals, Inc. Prescribing information for Rhopressa. Available from: https:/www.accessdata.fda.gov/ drugsatfda_docs/label/2017/208254lbl.pdf. ${ }^{33}$ are present in higher amounts in the TM when compared to the ciliary muscle of human eyes; therefore, the main effect of IOP regulation is thought to be through this effect on the TM. ${ }^{12,14}$ In a study by Wang et al, ${ }^{15}$ monkey eyes dosed with $0.04 \%$ netarsudil had significantly increased trabecular outflow facility by $53 \%$ when compared to contralateral vehicle-treated or baseline eyes $(0.90 \pm 0.11$ vs $0.58 \pm .07 \mu \mathrm{L} / \mathrm{min} / \mathrm{mmHg}$ at baseline, $P<0.05)$. Netarsudil additionally reduces IOP by decreasing episcleral venous pressure and decreasing the production of aqueous humor. ${ }^{15,16}$

\section{Efficacy in animal studies}

Netarsudil has been shown to be effective in IOP reduction in early phase animal studies. Lin et $\mathrm{al}^{8}$ determined a dosedependent relationship when using netarsudil, in which netarsudil $0.04 \%$ demonstrated a significantly greater IOP reduction compared to doses of $0.005 \%$ in rabbits only and $0.01 \%$ and $0.02 \%$ in rabbits and monkeys $(P<0.05)$. The study also showed that netarsudil $0.04 \%$ demonstrated a significantly greater reduction in IOP when compared to an earlier stage ROCK-selective compound (AR-12286 0.5\% Ophthalmic Solution) with maximal IOP reductions on Day 3 of therapy of $8.1 \pm 0.7$ and $7.5 \pm 1.1 \mathrm{mmHg}$ in rabbits and monkeys, respectively ( $P<0.01$ for both). ${ }^{8}$ In another study done by Li et a ${ }^{17}$ using $0.04 \%$ netarsudil, an average IOP reduction of $5.2 \mathrm{mmHg}$ was observed when compared to the contralateral untreated eye in mice.

\section{Review of clinical results}

The efficacy of netarsudil has been compared to both timolol and latanoprost in clinical trials. In two double-masked, randomized clinical trials called Rho Kinase Elevated IOP Treatment Trial 1 (ROCKET-1) and Rho Kinase Elevated IOP Treatment Trial 1 (ROCKET-2), Serle et al ${ }^{18}$ observed clinically relevant and statistically significant IOP reductions in netarsudil $0.02 \%$ solution dosed once daily at night (QHS) when compared to timolol twice a day (BID) in patients with a maximum baseline IOP $<25 \mathrm{mmHg}$ (Table 1). The two Phase III FDA-registration trials enrolled 1,167 patients, with the ROCKET-2 trial enlisting an additional study arm randomized to BID dosing of netarsudil. After an appropriate washout of preexisting IOP-lowering medicines, patients were randomized using a computer-generated method. Unmedicated IOP was required to be between 20 and $27 \mathrm{mmHg}$ for study inclusion. Eyes with secondary openangle glaucomas or occludable anterior chamber angles were excluded from the study. Netarsudil noninferiority to timolol was prospectively determined to be met if the upper limit 
Table I Efficacy results with netarsudil $0.02 \%$ daily dosing in clinical trials

\begin{tabular}{lllll}
\hline $\begin{array}{l}\text { Clinical } \\
\text { trial }^{\mathrm{a}}\end{array}$ & $\begin{array}{l}\text { Sample } \\
\text { size }\end{array}$ & $\begin{array}{l}\text { Mean baseline } \\
\text { IOP }(\mathbf{m m H g})\end{array}$ & $\begin{array}{l}\text { Mean 3-month } \\
\text { IOP (8 AM) }\end{array}$ & $\begin{array}{l}\text { Noninferiority } \\
\text { to timolol 0.5\% } \\
\text { twice daily? }\end{array}$ \\
\hline ROCKET-I $^{\mathrm{b}}$ & 182 & 23.42 & 19.81 & No \\
ROCKET-I $^{\mathrm{c}}$ & 113 & 22.39 & 18.22 & Yes \\
ROCKET-2 $^{\mathrm{d}}$ & 129 & 22.54 & 18.24 & Yes \\
\hline
\end{tabular}

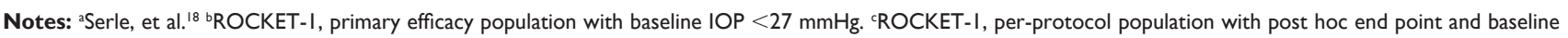

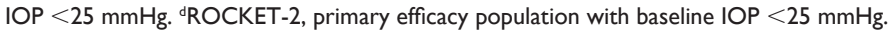

Abbreviations: IOP, intraocular pressure; ROCKET-I, Rho Kinase Elevated IOP Treatment Trial I; ROCKET-2, Rho Kinase Elevated IOP Treatment Trial 2.

of the two-sided CI around the difference in IOP lowering was within $1.5 \mathrm{mmHg}$ at 8:00 $\mathrm{AM}, 10: 00 \mathrm{AM}$, and 4:00 PM time points at week 2 , week 6 , and month 3 visits post randomization. The treatment arm randomized to netarsudil $0.02 \%$ QHS experienced a clinically relevant decrease in IOP at $8 \mathrm{AM}, 10 \mathrm{AM}$, and $4 \mathrm{PM}$ time points on the 3-month visit. Using a post hoc end point (for the ROCKET-1 trial) in patients with baseline IOP $<25 \mathrm{mmHg}$, netarsudil met noninferiority criteria when compared to timolol at the $8 \mathrm{AM}$ (18.22 vs $17.91 \mathrm{mmHg}$, respectively; two-sided $95 \% \mathrm{CI}= \pm 1.02 \mathrm{mmHg}$ ), $10 \mathrm{AM}$ (17.34 vs $17.43 \mathrm{mmHg}$, respectively; two-sided $95 \% \mathrm{CI}= \pm 0.63 \mathrm{mmHg}$ ), and 4 PM (17.02 vs $17.37 \mathrm{mmHg}$, respectively; two-sided 95\% $\mathrm{CI}= \pm 0.34 \mathrm{mmHg}$ ) time points. However, netarsudil $0.02 \%$ QHS did not meet the non-inferiority criteria when compared to timolol in the ROCKET-1 trial when the baseline IOP was set at $<27 \mathrm{mmHg}$ in primary analysis based on an IOP-lowering difference $>1.5 \mathrm{mmHg}$ at the $8 \mathrm{AM}$ time point at week 6 (19.35 vs $18.24 \mathrm{mmHg}$, respectively; two-sided $95 \% \mathrm{CI}= \pm 1.80 \mathrm{mmHg}$ ) and the $8 \mathrm{AM}(19.81$ vs $18.47 \mathrm{mmHg}$, respectively; $95 \% \mathrm{CI}= \pm 2.03 \mathrm{mmHg}$ ) and $10 \mathrm{AM}(18.92 \mathrm{vs}$ $17.96 \mathrm{mmHg}$, respectively; $95 \% \mathrm{CI}= \pm 1.66 \mathrm{mmHg}$ ) time points at month 3. Serle et $\mathrm{al}^{18}$ hypothesized that perhaps patients selected for this unmedicated baseline IOP did not maintain the observed baseline outside of the day of enrollment and instead maintained higher unmedicated baseline IOPs.

When compared to latanoprost, a study performed by Bacharach et a ${ }^{19}$ showed that $0.01 \%$ netarsudil, $0.02 \%$ netarsudil, and latanoprost patient groups experienced clinically relevant IOP reductions of 5.5, 5.7, and $6.8 \mathrm{mmHg}$, respectively, from an unmedicated baseline $(P<0.001)$. Despite these reductions, both $0.01 \%$ and $0.02 \%$ netarsudil once again did not meet the predetermined noninferiority criteria, this time against latanoprost. ${ }^{19}$ However, Bacharach et al ${ }^{19}$ did observe that netarsudil had similar efficacy to latanoprost when observed in a prespecified subgroup of patients with a baseline IOP $<26 \mathrm{mmHg}$.
In these studies, the importance of baseline IOP when determining the noninferiority of netarsudil should not be overlooked. In both the timolol and latanoprost studies, baseline IOP dictated whether a significant IOP reduction when using netarsudil was comparable to that of the other medication in that study. ${ }^{18,19}$

\section{Adverse effects}

The most frequently reported adverse effect in netarsudil studies was the presence of conjunctival hyperemia. ${ }^{19-21}$ This side effect is likely due to ROCK-mediated inhibition of calcium sensitization, resulting in the relaxation of blood vessel smooth muscle on the conjunctiva. ${ }^{13,22}$ The conjunctival hyperemia was mainly asymptomatic but was seen at higher rates in netarsudil when compared to other medications. In the ROCKET-1 and ROCKET-2 trials, timolol conjunctival hyperemia had an incidence of $8 \%-10 \%$ compared to netarsudil QHS at 50\%-53\% and netarsudil BID at 59\%. ${ }^{18}$ Bacharach et a ${ }^{19}$ also observed the conjunctival hyperemia at a similar rate of $52 \%$ and $57 \%$ for netarsudil $0.01 \%$ and $0.02 \%$ patients, respectively, vs the latanoprost group that had an incidence of $16 \%(P<0.001)$. However, the study of Bacharach et al ${ }^{19}$ did note that the frequency of conjunctival hyperemia decreased for netarsudil throughout the study period of 28 days. Additional longitudinal studies would be necessary in order to observe whether this decline in hyperemia continues to fade with time.

The next most frequent adverse effect observed was conjunctival hemorrhage. The ROCKET-1 and ROCKET-2 trials observed a $13.5 \%-15 \%$ incidence in netarsudil QHS, $17 \%$ incidence in netarsudil BID, and $0 \%-0.5 \%$ incidence in timolol patients. ${ }^{18}$ Conjunctival hemorrhage was also observed in 5\% of the combined netarsudil $0.01 \%$ and $0.02 \%$ groups in the study of Bacharach et al. ${ }^{19}$ PG324 0.01\% and $0.02 \%$, a solution composed of both netarsudil and latanoprost, also exhibited conjunctival hemorrhage when latanoprost alone did not. ${ }^{20}$ 
Additional less prominent adverse effects were observed, including mild corneal staining, headaches, blurry vision, instillation site pain/erythema, and eyelid erythema. ${ }^{18,20,21}$ No studies noted clinically significant netarsudil-related systemic adverse events. ${ }^{18,20,21}$ However, in the Lewis et al study, ${ }^{20}$ there was a greater number of discontinuations in patients taking netarsudil $0.02 \%$ BID likely due to the conjunctival hyperemia. Because more prominent hyperemia was observed during the day in netarsudil $0.02 \%$ BID patients, Serle et $\mathrm{al}^{18}$ suggested that QHS dosing would decrease the likelihood of symptoms during waking hours.

\section{Potential non-IOP lowering effects}

Netarsudil is regarded for its IOP-lowering effects; however, previous animal studies on other ROCK inhibitors, such as fasudil and ripasudil, have demonstrated potential benefits of agents in this class. ${ }^{23,24}$ These potential benefits include neuroprotection of the optic nerve head (ONH), improved ophthalmic perfusion, mitigation of inflammation, prevention of scarring post glaucoma filtration procedures, and promotion of corneal healing. ${ }^{23}$

Sugiyama et $\mathrm{al}^{24}$ assessed ocular blood flow at the $\mathrm{ONH}$ after treatment with fasudil. Responses to the agent were evaluated in rabbits with normal vascular flow in comparison to rabbits with vasoconstriction due to pretreatment with endothelin-1 and NG-nitro-L-arginine methyl ester. ${ }^{24}$ Subjects in the latter group showed improvement in $\mathrm{ONH}$ vascular flow upon treatment with fasudil. ROCK inhibitors function to relax vascular smooth muscle through either desensitization of $\mathrm{Ca}^{2+}$ receptors in contractile proteins or by induction of functional changes in Rho kinases. ${ }^{23,24}$ Enhanced blood flow to the ONH protects against its degeneration, attenuating the risk of optic neuropathy in patients with glaucoma. $^{24}$

Furthermore, ROCK inhibitors may confer neuroprotection by hindering the effects of RhoA and ROCKII that normally cause retinal cell ganglia damage via NMDA activation. ${ }^{25}$ Kitaoka et $\mathrm{al}^{25}$ reported these aforementioned findings in their study that involved administration of fasudil on a rat model they developed. In another study led by Sagawa et al, ${ }^{26}$ retinal axonal regeneration was demonstrated following administration of ROCK inhibitor Y-39983 injections at the site of optic nerve crush injury; treatment was effective up to a month following the initial damage. Moreover, there is evidence that ROCK inhibitors modify oxidative stress through downregulation of ROS production, protecting against disruption of the TM and maintaining a patent outflow pathway for the aqueous humor. ${ }^{27}$ Fujimoto et $\mathrm{al}^{27}$ identified these antioxidative effects at the level of the TM following the administration of ROCK inhibitor Y-27632 to monkey subjects. Overall, these therapeutic advantages have been primarily demonstrated in animal models. ${ }^{23-27}$ Further research exploring the spectrum of ROCK inhibitor functions in human populations is warranted. ${ }^{23}$ In addition, these non-IOP-lowering benefits are subjected to evaluation in netarsudil, in particular given its novelty.

\section{Clinical implications}

Inconsistent or poor glaucoma medication adherence is a risk factor for glaucoma progression, and medication compliance has been shown to be inversely related to the frequency of required dose per day. ${ }^{28,29}$ With a longer duration of action and once-daily dosing schedule, netarsudil may decrease risk of medication nonadherence. Furthermore, netarsudil's novel mechanism of action may allow for IOP-lowering efficacy in patients with suboptimal response to other available agents. ${ }^{30}$

In clinical trials, netarsudil application resulted in no observed serious systemic effects, but symptoms of conjunctival hyperemia may still potentially cause patient dissatisfaction. ${ }^{18,21}$ Netarsudil $0.02 \%$ BID led to a $30 \%$ discontinuation rate due to adverse events in the ROCKET-2 trial. ${ }^{18}$ However, only two of 147 patients taking netarsudil $0.01 \%$ and $0.02 \%$ QD required discontinuation due to adverse events in the study conducted by Bacharach et al. ${ }^{19}$ Bacharach et al also observed a difference in the patient-reported redness with night-time application of netarsudil vs the biomicroscopic observations at the clinic the next day. ${ }^{19}$ This implies that netarsudil symptoms decrease with time, and application at night allows patients to experience the worst of the symptoms while asleep. The study of Bacharach et $\mathrm{al}^{19}$ was only 28 days long, but a decrease in the incidence of conjunctival hyperemia was also observed throughout the length of the study. Long-term results should be observed, but there is also potential for a decrease in frequency of conjunctival hyperemia with continued use of netarsudil.

\section{Potential advantages for normal- tension glaucoma patients}

Netarsudil has potential use for normotensive patients managing glaucoma progression. The study of Wang et $\mathrm{al}^{15}$ on normotensive monkeys exhibited a $24 \%$ decrease in IOP with $0.04 \%$ netarsudil administration when compared to contralateral vehicle-treated eyes. The mechanism of action was linked to a significant $(P<0.05)$ increase in baseline outflow facility and decrease in aqueous flow rate with administration 
of netarsudil. ${ }^{15}$ In additional studies, netarsudil maintained efficacy of IOP reduction, regardless of baseline IOP. ${ }^{18,19}$ Latanoprost, used as a comparison medication in the study of Bacharach et al, ${ }^{19}$ more effectively lowered IOP in patients with a higher IOP baseline. In a Phase I study performed by Levy et al, ${ }^{21}$ normotensive patients with baseline IOPs between 14 and $20 \mathrm{mmHg}$ showed significant reductions over 8 days of $0.02 \%$ netarsudil use. These studies suggest that IOP reduction via netarsudil does not depend on the baseline IOP as it does for other glaucoma medications. Therefore, in patients who require an IOP below normal in order to stunt glaucoma progression, netarsudil may be an effective treatment option.

\section{Potential advantages for management of steroid-induced glaucomas}

Steroid-induced glaucoma is an iatrogenic disease process that parallels open-angle glaucoma and may be managed with a ROCK inhibitor. ${ }^{31,32}$ The mechanism behind this aforementioned type of glaucoma is through the noncanonical Wnt pathway that is coupled with ROR2/RhoA/ROCK. ${ }^{31}$ Yuan et $\mathrm{al}^{31}$ determined that administration of dexamethasone activates this pathway, promoting cross-linking of actin networks and causing architectural changes to the cytoskeleton of the TM. This ultimately leads to obstruction in the outflow of aqueous humor and elevation of IOP, which is a salient factor in the pathogenesis of glaucoma. Given netarsudil's key function of ROCK inhibition, it may be a viable treatment option for steroid-induced glaucomas.

\section{Potential for netarsudil and latanoprost combination}

There is also potential for a combination agent composed of netarsudil and latanoprost, currently referred to as PG324. In early studies, PG324 $0.02 \%$ resulted in a favorable mean IOP difference ranging from 1.6 to $3.2 \mathrm{mmHg}$ vs latanoprost and 1.7-3.4 mmHg vs AR-13324. ${ }^{20}$ As discussed earlier, mild asymptomatic conjunctival hyperemia was observed with application of PG324 0.01\%, PG324 0.02\%, latanoprost, and netarsudil groups at an incidence of $41 \%, 40 \%, 14 \%$, and $40 \%$, respectively, suggesting that these symptoms do not increase in frequency when using the combination of medications. $^{20}$

\section{Conclusion}

Netarsudil has a unique mechanism of action via increased trabecular outflow facility as well as decreased episcleral venous pressure and production of aqueous humor, which may allow for application of the medication in patient populations that have traditionally been more difficult to treat. ${ }^{15,16}$ However, though netarsudil has exhibited a significant reduction in IOP regardless of baseline, it has still failed the noninferiority tests when compared to both timolol and latanoprost. ${ }^{17,19}$ Therefore, it is unlikely to become a firstline medication at this time. Netarsudil does have a notable adverse effect of conjunctival hyperemia, although this side effect may potentially be managed through administration once in the evening before bedtime and may fade in intensity with time. ${ }^{18-21}$ Overall, netarsudil may be especially beneficial to patients suffering from normotensive or steroid-induced glaucoma. ${ }^{15,18,19,31,32}$

\section{Disclosure}

The authors report no conflicts of interest in this work.

\section{References}

1. Tham YC, Li X, Wong TY, Quigley HA, Aung T, Cheng CY. Global prevalence of glaucoma and projections of glaucoma burden through 2040: a systematic review and meta-analysis. Ophthalmology. 2014; 121(11):2081-2090.

2. Nemesure B, Honkanen R, Hennis A, Wu SY, Leske MC; Barbados Eye Studies Group. Incident open-angle glaucoma and intraocular pressure. Ophthalmology. 2007;114(10):1810-1815.

3. Schwartz K, Budenz D. Current management of glaucoma. Curr Opin Ophthalmol. 2004;15(2):119-126[pii].

4. Weinreb RN, Khaw PT. Primary open-angle glaucoma. Lancet. 2004; 363(9422):1711-1720.

5. Rao PV, Pattabiraman PP, Kopczynski C. Role of the Rho GTPase/Rho kinase signaling pathway in pathogenesis and treatment of glaucoma: Bench to bedside research. Exp Eye Res. 2017;158:23-32.

6. Bucolo C, Salomone S, Drago F, Reibaldi M, Longo A, Uva MG. Pharmacological management of ocular hypertension: current approaches and future prospective. Curr Opin Pharmacol. 2013;13(1):50-55.

7. Sambhara D, Aref AA. Glaucoma management: relative value and place in therapy of available drug treatments. Ther Adv Chronic Dis. 2014; 5(1):30-43.

8. Lin CW, Sherman B, Moore LA, et al. Discovery and preclinical development of netarsudil, a novel ocular hypotensive agent for the treatment of glaucoma. J Ocul Pharmacol Ther. 2018;34(1-2):40-51.

9. Kass MA, Heuer DK, Higginbotham EJ, et al. The Ocular Hypertension Treatment Study: a randomized trial determines that topical ocular hypotensive medication delays or prevents the onset of primary openangle glaucoma. Arch Ophthalmol. 2002;120(6):30.

10. Choy M. Pharmaceutical Approval Update. P T. 2018;43(4): 205-227.

11. Sturdivant JM, Royalty SM, Lin CW, et al. Discovery of the ROCK inhibitor netarsudil for the treatment of open-angle glaucoma. Bioorg Med Chem Lett. 2016;26(10):2475-2480.

12. Nakajima E, Nakajima T, Minagawa Y, Shearer TR, Azuma M. Contribution of ROCK in contraction of trabecular meshwork: proposed mechanism for regulating aqueous outflow in monkey and human eyes. J Pharm Sci. 2005;94(4):701-708.

13. Ren R, Li G, Le TD, Kopczynski C, Stamer WD, Gong H. Netarsudil increases outflow facility in human eyes through multiple mechanisms. Invest Ophthalmol Vis Sci. 2016;57(14):6197-6209.

14. Rao VP, Epstein DL. Rho GTPase/Rho kinase inhibition as a novel target for the treatment of glaucoma. BioDrugs. 2007;21(3):167-177. 
15. Wang RF, Williamson JE, Kopczynski C, Serle JB. Effect of 0.04\% AR-13324, a ROCK, and norepinephrine transporter inhibitor, on aqueous humor dynamics in normotensive monkey eyes. J Glaucoma. 2015;24(1):51-54.

16. Kiel JW, Kopczynski CC. Effect of AR-13324 on episcleral venous pressure in Dutch belted rabbits. J Ocul Pharmacol Ther. 2015; 31(3):146-151.

17. Li G, Mukherjee D, Navarro I, et al. Visualization of conventional outflow tissue responses to netarsudil in living mouse eyes. Eur $J$ Pharmacol. 2016;787:20-31.

18. Serle JB, Katz LJ, McLaurin E, et al. Two phase 3 clinical trials comparing the safety and efficacy of netarsudil to timolol in patients with elevated intraocular pressure: Rho kinase elevated IOP treatment trial 1 and 2 (ROCKET-1 and ROCKET-2). Am J Ophthalmol. 2018;186:116-127.

19. Bacharach J, Dubiner HB, Levy B, Kopczynski CC, Novack GD, AR-13324-CS202 Study Group. Double-masked, randomized, doseresponse study of AR-13324 versus latanoprost in patients with elevated intraocular pressure. Ophthalmology. 2015;122(2):302-307.

20. Lewis RA, Levy B, Ramirez N, et al. Fixed-dose combination of AR-13324 and latanoprost: A double-masked 28-day, randomised, controlled study in patients with open-angle glaucoma or ocular hypertension. [- 3]. (- 1468-2079 (Electronic); - 0007-1161 (Linking)).

21. Levy B, Ramirez N, Novack GD, Kopczynski C. Ocular hypotensive safety and systemic absorption of AR-13324 ophthalmic solution in normal volunteers. Am J Ophthalmol. 2015;159(5):5.e1.

22. Uehata M, Ishizaki T, Satoh H, et al. Calcium sensitization of smooth muscle mediated by a Rho-associated protein kinase in hypertension. Nature. 1997;389(6654):990-994.

23. Honjo M, Tanihara H. Impact of the clinical use of ROCK inhibitor on the pathogenesis and treatment of glaucoma. Jpn J Ophthalmol. 2018;62(2):109-126.

24. Sugiyama T, Shibata M, Kajiura S, et al. Effects of fasudil, a Rhoassociated protein kinase inhibitor, on optic nerve head blood flow in rabbits. Invest Ophthalmol Vis Sci. 2011;52(1):64-69.
25. Kitaoka Y, Kitaoka Y, Kumai T, et al. Involvement of RhoA and possible neuroprotective effect of fasudil, a Rho kinase inhibitor, in NMDA-induced neurotoxicity in the rat retina. Brain Res. 2004;1018(1): 111-118.

26. Sagawa H, Terasaki H, Nakamura M, et al. A novel ROCK inhibitor, Y-39983, promotes regeneration of crushed axons of retinal ganglion cells into the optic nerve of adult cats. Exp Neurol. 2007;205(1): 230-240[pii].

27. Fujimoto $\mathrm{T}$, Inoue $\mathrm{T}$, Ohira $\mathrm{S}$, et al. Inhibition of rho kinase induces antioxidative molecules and suppresses reactive oxidative species in trabecular meshwork cells. J Ophthalmol. 2017, Article ID 7598140, 23 pages.

28. Sleath B, Blalock S, Covert D, et al. The relationship between glaucoma medication adherence, eye drop technique, and visual field defect severity. Ophthalmology. 2011;118(12):2398-2402. https://www. clinicalkey.es/playcontent/1-s2.0-S0161642011004568.

29. Claxton AJ, Cramer J, Pierce C. A systematic review of the associations between dose regimens and medication compliance. Clin Ther. 2001;23(8):1296-1310[pii].

30. Tanna AP, Johnson M. Rho Kinase Inhibitors as a Novel Treatment for Glaucoma and Ocular Hypertension. Ophthalmology. 2018 Jul 11. (Epub ahead of print).

31. Yuan Y, Call MK, Yuan Y, et al. Dexamethasone induces cross-linked actin networks in trabecular meshwork cells through noncanonical wnt signaling. Invest Ophthalmol Vis Sci. 2013;54(10):6502-6509.

32. Fujimoto $T$, Inoue $T$, Kameda $T$, et al. Involvement of RhoA/Rhoassociated kinase signal transduction pathway in dexamethasoneinduced alterations in aqueous outflow. Invest Ophthalmol Vis Sci. 2012; 53(11):7097-7108.

33. Aerie Pharmaceuticals, Inc. Prescribing information for Rhopressa. Available from: https://www.accessdata.fda.gov/drugsatfda_docs/ label/2017/208254lbl.pdf. Accessed September 26, 2018.
Clinical Ophthalmology

\section{Publish your work in this journal}

Clinical Ophthalmology is an international, peer-reviewed journal covering all subspecialties within ophthalmology. Key topics include: Optometry; Visual science; Pharmacology and drug therapy in eye diseases; Basic Sciences; Primary and Secondary eye care; Patient Safety and Quality of Care Improvements. This journal is indexed on Submit your manuscript here: http://www.dovepress.com/clinical-ophthalmology-journal

\section{Dovepress}

PubMed Central and CAS, and is the official journal of The Society of Clinical Ophthalmology (SCO). The manuscript management system is completely online and includes a very quick and fair peer-review system, which is all easy to use. Visit http://www.dovepress.com/ testimonials.php to read real quotes from published authors. 